\title{
Exploration of User Separation Capabilities by Distributed Large Antenna Arrays
}

\author{
Cheng-Ming Chen, Vladimir Volskiy, Alessandro Chiumento, \\ Liesbet Van der Perre, Guy A. E. Vandenbosch and Sofie Pollin \\ Departement of Electrical Engineering, KU Leuven, Belgium \\ Email: \{cchen,volski,achiumen,lvanderp,vdbosch,spollin\}@esat.kuleuven.be
}

\begin{abstract}
In this paper, we present a novel exploration of spatial separation of closely-located users by a massive MIMO system in line-of-sight (LoS) for both an anechoic chamber and an indoor corridor propagation environments. A distributed massive multiple-input multiple-output (DM-MIMO) system powered by software defined radios (SDRs) has been used for the measurement campaign. For the user separation study, we first conduct an analysis with a spherical wave channel model for physically-large rectangular arrays. Preliminary simulation results show that a single antenna array achieves better user separation for users distributed on a line parallel to the array plane rather than when users are distributed on a line normal to the array. An intuitive idea is that, by splitting a collocated antenna array into two subarrays, additional diversity is created if the second sub-array is on a plane orthogonal to the first sub-array. Figures of merit such as the correlation coefficients, condition numbers and sum-rates of both centralized and distributed antenna arrays have been chosen to determine the impact of array split and positioning on user separation. Extensive measurements, performed on two 32element antenna arrays of a massive MIMO testbed at $2.6 \mathrm{GHz}$, show that an improvement of around $140 \%$ can be achieved in a poor scattered environment if distributed arrays are used instead of centralized ones.
\end{abstract}

Index Terms-large-scale antenna systems, massive MIMO, spherical wave model, distributed antenna array, spatial separation, software defined radio

\section{INTRODUCTION}

With massive MIMO, a base station (BS) employs a large number of antennas and sends independent data streams to multiple terminals in the same time and frequency unit, therefore the spectral efficiency is greatly enhanced [1]-[3]. Through the large number of degrees-of-freedom available for each user equipment (UE), under "favorable propagation conditions", massive MIMO provides high performance and reliability by a very simple linear processing such as matchedfiltering (MF) or zero-forcing (ZF) [1], [2]. However, if the channel vectors among the users are highly correlated, favorable conditions do not exist and the overall channel capacity and transmit energy efficiency degrade significantly [3], [4].

Much research has been conducted to study realistic massive MIMO channel characteristics. In [5], the authors conclude that the near-field effects and the non-stationarities over the array help decorrelating channels of different UEs. Both [4], [6] tried to improve user separation by means of increasing the number of antennas in massive MIMO. The UE deployment considered in [6] was fairly random and thus not limited to closely deployed users. When using a horizontal antenna array, the authors conclude that by increasing the number of antennas, a further reduction of the correlation coefficient between two UEs can be expected, nevertheless, this cannot be directly applied to the case when the users are closely located. In [4], the authors however focused on the user separation of closely-located UEs with a cross-polarized cylindrical antenna array at the BS. There is little improvement in the closely distributed user separation when increasing the number of antennas from 64 to 128 . Furthermore, from the results of the work, there is no strong indication that a collocated antenna array performs well in closely located user separation. For instance, there is still a huge gap for the distribution of the condition numbers between ideal independent identically distributed (i.i.d.) and measured channels under 128 antennas.

In this paper, instead of increasing the number of antennas, we aim to decorrelate closely spaced UEs by distributing the antennas into multiple arrays. For UEs located inside the nearfield (in the case of our collocated antenna array, the range is around $11.8 \mathrm{~m}$ ), the spherical wave helps to decorrelate users even in LoS scenarios [10]. Nonetheless, when users are spatially close to each other or there is little variation in the AoAs from the UEs to the antenna array, user separation becomes challenging. The difference of this work to [4], is that instead of restricting UEs randomly distributed within a circle of 5 meters in radius we investigate the capability of user separation when users are distributed in the saturation region. A saturation region denotes a specific region where user correlation is not significantly improved when increasing distance among the UEs. First, an analytical channel model for a physically large rectangular array, which takes spherical wavefronts into consideration is employed to find this region. We show that in the near-field, the saturation region is when users are located on a line normal to the array, and the region where best user separation is achieved is when users are located on a line parallel to the array. Then, from measurements, we show that antenna separation in the same 2D plane does not improve user separation, but having the second sub-array in a plane orthogonal to the first sub-array hugely improves system performance. Here we conducted the measurements in two scenarios, in an extreme scenario like an anechoic chamber, where only LoS paths contribute to 
the propagation channel, the measurement results represent a very good reference for any poor-scattering environment. Moreover, a narrow indoor corridor is considered as an alternative comparison to the previous scenario, so when more multipaths are taken into account, it is of interest to know how much improvement will be observed in the user separation. To the best of our knowledge, this is the first paper reporting such distributed large-scale antenna channel measurements. The paper is organized as follows. Section II describes the system model and the relevant performance metrics. Section III presents the spherical wave channel model of large scale rectangular arrays. The setup of the SDR testbed for distributed channel measurement is introduced in section IV. The results of the paper are summarized in section $\mathrm{V}$ and concluded in section VI.

\section{Signal Model And Performance Metrics}

A downlink single-cell MU-MIMO system is considered in this paper, where a BS with large number of antennas $M$ communicates with $K(K \leq M)$ single-antenna UEs. The communication is then modulated with orthogonal frequency division multiplexing (OFDM). Vector $\mathbf{h}^{T}{ }_{k, n, t}$ represents the $k$ th row of the channel matrix $\mathbf{H}_{n, t} \in \mathbb{C}^{K \times M}$ at subcarrier $n$ and snapshot $t$, with $1 \leq n \leq N$ and $1 \leq t \leq T$, satisfying a fixed long-term power for each UE

$$
\sum_{t=1}^{T} \sum_{n=1}^{N}\left\|\mathbf{h}_{k, n, t}\right\|_{F}^{2}=T N
$$

where $N$ is the total number of subcarriers, $T$ is the total number of measurment snapshots and $\|\cdot\|_{F}$ is the Frobenius norm. The power constraint applied here is a normalization procedure to compensate for gain imbalances across different MISO links. For notational simplicity, we ignore the dependence of $\mathbf{H}$ on $n$ and $t$. Let $\mathbf{s} \in \mathbb{C}^{M \times 1}$ be the vector signal transmitted by the BS simultaneously to all $K$ UEs, it satisfies the sum power constraint

$$
\mathbb{E}\left[\|\mathbf{s}\|_{F}^{2}\right]=1
$$

The vector of the received signals $\mathbf{y} \in \mathbb{C}^{K \times 1}$ at the $\mathrm{K}$ UEs is given as

$$
\mathbf{y}=\sqrt{\rho} \mathbf{H} \mathbf{s}+\mathbf{n},
$$

where $\rho$ is the signal to noise ratio (SNR) of the link, it is proportional to the transmitted power divided by the noisevariance $\mathbf{n} \sim \mathcal{C N}\left(\mathbf{0}, K^{-1} \mathbf{I}_{K}\right)$ which is an i.i.d. circularysymmetric complex Gaussian distributed noise vector.

In order to quantify the degree of orthogonality among the UEs, three different performance indicators are used. First, the correlation coefficient is calculated as it checks the correlation pair-wisely between two UEs and is thus useful to determine the separation of the users with respect to each other. However, for a massive MIMO system, more than two UEs are serviced simultaneously, thus, it is indeed relevant to look at a joint orthogonality indicator by looking into the singular value spread. To avoid looking at the equivalent channels on only the best and the worst streams, we also consider the sum-rates of ZF linear precoding [7].

\section{A. Correlation Coefficient}

The correlation coefficient between the channel vectors of two UEs $k_{1}$ and $k_{2}$ is defined as

$$
\delta_{k_{1}, k_{2}}=\frac{\left|\mathbf{h}_{k_{1}}^{H} \mathbf{h}_{k_{2}}\right|}{\left\|\mathbf{h}_{k_{1}}\right\|_{F}\left\|\mathbf{h}_{k_{2}}\right\|_{F}},
$$

where (.) ${ }^{H}$ denotes the Hermitian transpose and $0 \leq$ $\delta_{k_{1}, k_{2}} \leq 1$. The correlation coefficient evaluates the "orthogonality" of the pair-wise channel vectors.

\section{B. Singular Value Spread (Condition Number)}

The singular value spread of the Gramian matrix $\mathbf{G}=$ $\mathbf{H H}^{H}$ is introduced here as a measure of the degree of orthogonality among all the UEs.

$$
\kappa(d B)=10 \log _{10}\left(\frac{\sigma_{\max }(\mathbf{G})}{\sigma_{\min }(\mathbf{G})}\right),
$$

where $\sigma_{\max }($.$) and \sigma_{\min }($.$) are the maximum and mini-$ mum singular values of a matrix and $\kappa=[1, \infty)$. A lower $\kappa$ means a more favorable conditioned channel for multi-user communication [8].

\section{Sum-Rates}

The precoded vector $\mathbf{s}$ is of the form

$$
\mathbf{s}=\mathbf{W} \mathbf{x} .
$$

The vector $\mathbf{x}$ comprises data symbols from an alphabet $\chi$, and each entry has unit average energy, i.e. $\mathbb{E}\left[\left\|x_{k}\right\|^{2}\right]=$ $1, k=1, \ldots, K$. ZF pre-coding eliminates the interference by transmitting the signals towards the intended user with nulls in the "direction" of other users. The precoding matrix is defined as

$$
\mathbf{W}_{Z F}=\mathbf{H}^{\dagger} \mathbf{P}_{Z F},
$$

where $\mathbf{H}^{\dagger}=\mathbf{H}^{H}\left(\mathbf{H} \mathbf{H}^{H}\right)^{-1}$ is the pseudoinverse of the channel matrix $\mathbf{H}$. An equal power for each UE after precoding is met after multiplying by the power allocation matrix $\mathbf{P}_{Z F}$

$$
\mathbf{P}_{Z F}=\left[\begin{array}{ccc}
\left(\left\|\mathbf{h}_{1}^{\dagger}\right\|_{F}\right)^{-1} & 0 & 0 \\
0 & \ddots & 0 \\
0 & 0 & \left(\left\|\mathbf{h}_{K}^{\dagger}\right\|_{F}\right)^{-1}
\end{array}\right]
$$

where $\mathbf{h}_{k}^{\dagger}$ is the $k_{t h}$ column of the pseudoinverse matrix $\mathbf{H}^{\dagger}$. We then collapse the product of the channel and the precoder matrices into a single matrix $\mathbf{A}=\mathbf{H W}$ for compactness. The achievable sum-rate $R_{\text {sum }}$ with linear precoding is then given as [7], [11] 


$$
R_{\text {sum }}=\sum_{k=1}^{K} \log _{2}\left(1+\gamma_{k}\right),
$$

where

$$
\gamma_{k}=\frac{\left[\operatorname{diag}(\mathbf{A}) \cdot \operatorname{diag}\left(\mathbf{A}^{H}\right)\right]_{k, k}}{\rho^{-1}},
$$

where $\operatorname{diag}(\mathbf{A})$ is a diagonal matrix containing only the principal diagonal of $\mathbf{A}$ and $[.]_{k, k}$ represents the $k_{t h}$ element of the principal diagonal of a matrix.

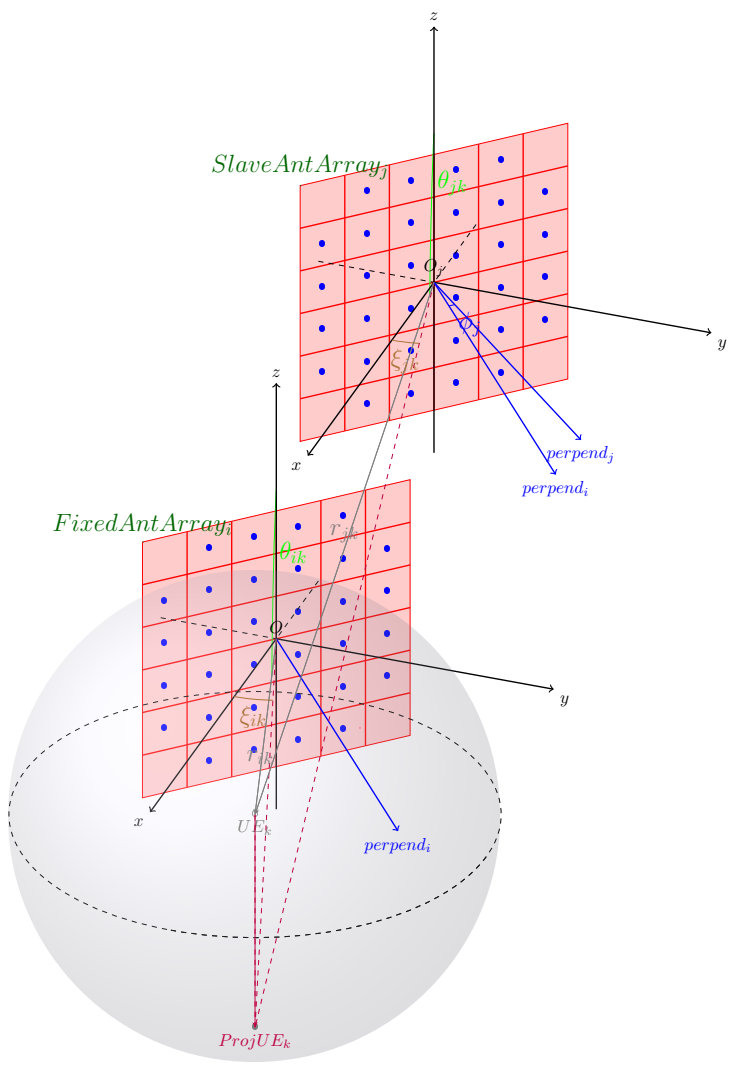

Fig. 1. Spherical wave channel model for distributed rectangular arrays

\section{Spherical Wave Channel Model for ReCtangular Large SCALE ARrays}

To investigate the user separation from a large scale antenna array in a near-field LoS scenario, a spherical wave massive MIMO channel model for rectangular arrays is introduced. There is no scattering in the free-space channel model thus no small-scale fading. Each UE and antenna element in the arrays are considered as a small source with an omni-directional radiation pattern, hence the superposition between them can be expressed in terms of spherical waves. The model further considers a distributed deployment of the large-scale antenna array. Concretely, our analysis assumes the case where the array is split into two antenna sub-arrays that can be freely located in the $x, y$ or $z$ plane. Fig. 1 portrays the coordinate system of the spherical wave model where total of $M$ antennas are split into a fixed and a slave arrays with equal number $\frac{M}{2}$ of antennas. The center of the array FixedAntArray is treated as the origin $O$ and the center of the slave array is positioned at the origin $O_{j}$. The normal vectors to the fixed and slave array panels are called perpend $d_{i}$ and perpend $d_{j}$ respectively and an azimuthal angle between the two is called $\varphi_{j}$ with $0 \leq \varphi_{j}<2 \pi$. $\delta_{r}$ is the normalized antenna spacing of the array in wavelength $\lambda_{c}$, and the coordinates of $k$ th UE location, $\mathrm{UE}_{k}$ can be represented by a spherical system (radial, azimuthal, zenith $)=\left(r_{i k}, \xi_{i k}, \theta_{i k}\right)$ referencing to the origin $O$. The coordinates of each antenna element can be determined by information of $\delta_{r} \lambda_{c}$ and the angles between the normal vectors to $\mathrm{x}$-axis. In this section, we assume the LoS is the only path in the channel. Hence, the spherical-wave eigenmode for the channel coefficient $h$ between the $\mathrm{UE}_{k}$ and any of the antenna elements is [10]

$$
h \propto \frac{\exp \left(-j \frac{2 \pi r}{\lambda_{c}}\right)}{r},
$$

where $r$ is the distance between the transmit and receive antenna. Finally, by calculating the distance $r_{k m}$ between $\mathrm{UE}_{k}$ to each antenna element $m \in\{1, \ldots, M\}$, the corresponding spherical wave channel vector can be expressed as

$\mathbf{h}_{k}^{T} \propto\left[\begin{array}{llll}\frac{\exp \left(-j \frac{2 \pi r_{k 1}}{\lambda_{c}}\right)}{r_{k 1}} & \frac{\exp \left(-j \frac{2 \pi r_{k 2}}{\lambda_{c}}\right)}{r_{k 2}} \quad \ldots \quad \frac{\exp \left(-j \frac{2 \pi r_{k M}}{\lambda_{c}}\right)}{r_{k M}} \quad(12)\end{array}\right]$.

We demonstrate that user distance is not the only factor that determines separation, but also the plane along which users are separated. A simple scenario is investigated by using the introduced channel model. As shown in Fig. 2, two groups of UEs are deployed, one lies on the line normal to the antenna plane, and the other one parallel. The correlation between the first UE to the other individual UEs on the two different lines is examined. The distance between the first UE to the origin $O$ of the fixed sub-array is $2 \mathrm{~m}$ and the distance between the first UE to the other UEs is increased in increments of $1 \mathrm{~m}$. As depicted in Fig. 3, there is a wider range of AoAs among the UEs to the antenna array when UEs are located on the line parallel to the antenna panel. The wide range of AoAs improves the ability of user separation. This observation suggests that we should deploy the slave sub-array in a plane perpendicular to the fixed sub-array with $\varphi_{j}=\frac{\pi}{2}$. We also notice that the correlation coefficient of the users deployed in parallel to the antenna panels grows up slightly when the UE moves further away from the first UE. There are two reasons for this phenomenon: first, the UE is moving in the direction to the far-field zone of the antenna array; and second the azimuthal angle between the vector $O \mathrm{UE}$ to the antenna panel approaches zero. Hence, the AoAs spread from the UE to the antenna array becomes fairly narrow. 


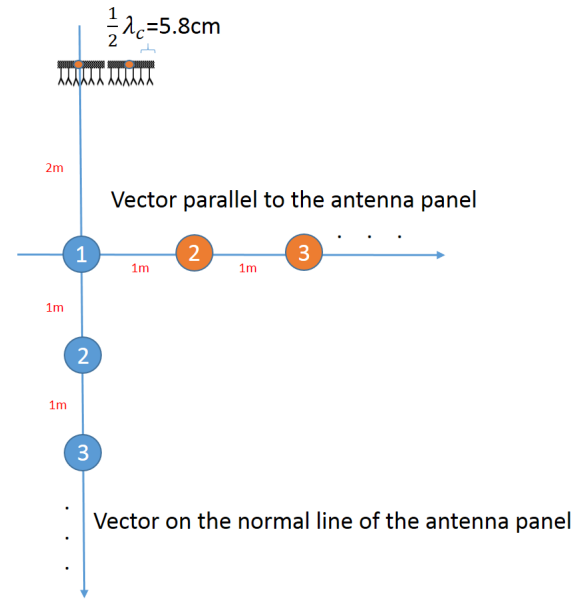

Fig. 2. The influence of UEs' distribution to the pair-wise correlation coefficient. A senario is designed and simulated with the theoretical model, there are two different UE distributions for their locations which is dependent on the relative direction of the antenna panel. Blue circles indicate UEs are located on the normal line of the antenna panel, and orange ones indicate UEs are located on the line parallel to the antenna panel. $\mathrm{UE}_{1}$ however is shared between the two groups of UEs.

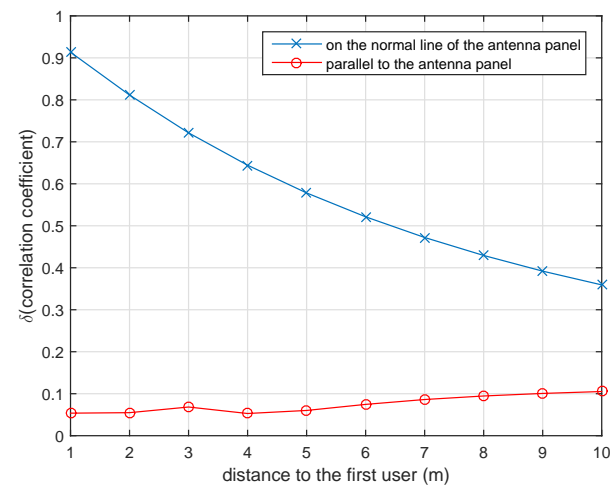

Fig. 3. Correlation coefficient comparison when UEs are on the line normal to the antenna panel vs. parallel to the antenna panel. If UEs distribute on the normal line of the antenna panel, as there is less AoA spread among the antennas, it is more challenge to separate the closely located UEs.

\section{Measurement Description}

\section{A. Measurement Setup}

Our measurement was conducted at $2.6 \mathrm{GHz}$ with $20 \mathrm{Mhz}$ bandwidth in a 802.11a OFDM system (subcarrier spacing equals to $312.5 \mathrm{kHz}$ ). At the UE side, we use a generic vertically-polarized omni-directional antenna and a singleinput and single-output (SISO) 802.11a system in the NI LabVIEW Communications 802.11 Application Framework [9] is running in a single SDR (NI 2942R/USRP RIO). In the measurement, we assume the uplink and downlink are perfectly reciprocal, hence the downlink channel for precoding is measured in uplink. We sequentially measure the channel between one UE to the whole BS antenna array, and stack all the row channel vectors from all UEs to a full downlink channel matrix. The set-up in the BS consists of two rectangular planar antenna arrays with a total of $M=64$ patch antennas, all of them were set in vertical polarization. Moreover, the massive MIMO prototype is built as an extension of the SISO 802.11a system to a 64 chains system and it is simultaneously running in 32 SDRs. There are two full-duplex RF bandwidth transceivers in each SDR and an independent over-the-air timing estimation algorithm is implemented in each receive chain. With the independent timing estimation, even if the sub-arrays are placed far away, there is no inter-symbolinterference (ISI) guaranteed in any of the antenna chains. The transmit power of the UE is $20 \mathrm{dBm}$, and in the receiver, a fixed gain of $37.5 \mathrm{~dB}$ is applied for all the antenna chains. Because the receiver gain is fixed for all antennas, the energy variations over BS antenna elements, subcarriers and measurement time snapshots are retained. Prior to data analysis, a channel vector normalization as in Eq. 1 is applied, so we have equal power for each MISO link. During our measurements, the frequency domain channel responses of the legacy long training field (L-LTF) with $N=52$ valid subcarriers were collected every $250 \mathrm{~ms}$ and a total of $T=80$ packets (snapshots in the system model) were collected for one measured UE position.

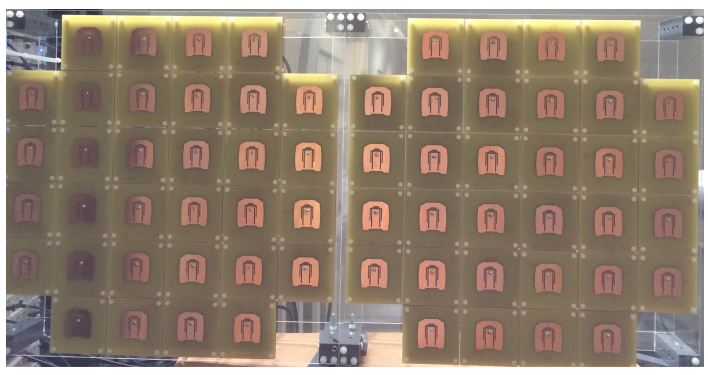

Fig. 4. Collocated rectangular planar antenna array

\section{B. Antenna}

The antenna element used in our BS is a patch antenna with two merged U-slots. This antenna topology allows a multiband performance covering $2.6 \mathrm{GHz}$ and $3.6 \mathrm{GHz}$ bands. The antenna element has a linear polarization and the realized gain is about $7 \mathrm{dBi}$ at $2.6 \mathrm{GHz}$. The $3 \mathrm{~dB}$ beam width in the E-plane and H-plane are about $72^{0}$ and $79^{0}$ respectively. Each element is fixed in one of 32 individual slots on a plexyglass panel with a spacing of $75 \mathrm{~mm}$ (close to half a wavelength at $2.6 \mathrm{GHz}$ ). Two panels can be used at the same time for channel measurements as shown in Fig. 4.

\section{Measurement Scenarios}

The channel measurements were conducted in an anechoic chamber and an indoor corridor of the ESAT building of KU Leuven as sketched in Fig. 5 and Fig. 6 respectively. The number of BS antennas in our system is 64 , with closely deployed UEs, we consider a low system load of roughly $10 \%$ so the number of 6 UEs is chosen. In both scenarios, a large antenna array with 64 elements is split into two subarrays with equal number of 32 antennas. $F$ represents the sub-array with a fixed position and there are 6 UEs placed on 


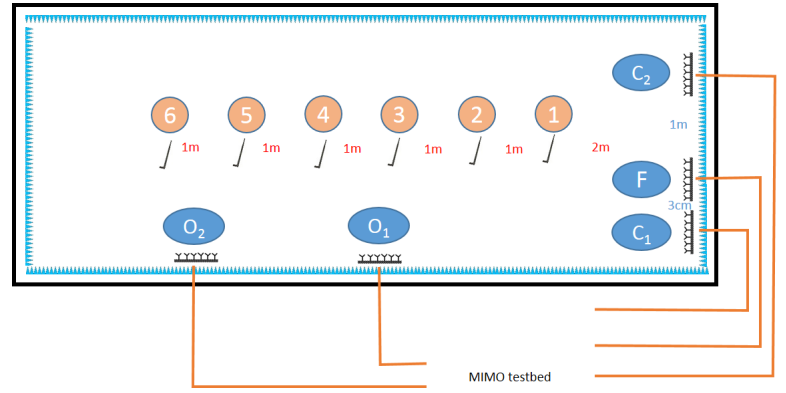

Fig. 5. Measurement environment in an anechoic chamber

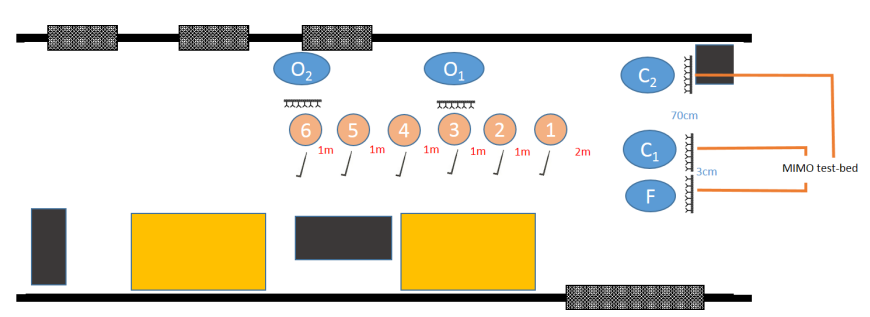

Fig. 6. Measurement environment in the indoor corridor

a line normal to the antenna panel $F$; the distance between the antenna panel and the first UE is $2 \mathrm{~m}$, then for every $1 \mathrm{~m}$ distance, we put the next UE. UEs are represented in numbers $1 \sim 6$ in the figures. For a collocated array case, the slave sub-array is labeled as $C_{1}$ with $3 \mathrm{~cm}$ separation from sub-array $F$. The slave sub-array is moved to the other three locations $\mathrm{C}_{2}, \mathrm{O}_{1}$ and $\mathrm{O}_{2}$ to consider three different distributed antenna arrays cases along two different planes. We define $C_{1}$ and $C_{2}$ here as co-directional antenna panels to the panel of $F$. Conversely, the normal lines of the other two sub-arrays $O_{1}$ and $\mathrm{O}_{2}$ are orthogonal to the normal line of antenna panel $F$.

\section{EXPERIMENTAL RESULTS AND ASSESSMENT}

In this section, we evaluate the user separation for massive MIMO, in particular, when the 6 UEs are located closely to each other and lay on the line perpendicular to the plane of the collocated antenna array panels. First, a plotting of the cumulative density function (CDF) of all snapshots and subcarriers of the condition numbers is presented in Fig. 7. We first look at the scenario of anechoic chamber and set the curve of this scenario $F+C_{1}$ as the benchmark. Obviously, there is little improvement in user separation with only a distance separation of antenna arrays $F+C_{2}$, this is because the slave antenna array is in co-direction to the fixed antenna array. However, if we deploy the slave antenna array to either position $\mathrm{O}_{1}$ or $\mathrm{O}_{2}$, as the slave antenna array lies on the line parallel to the user distribution, so there is around $10 d B$ left shift in condition number from curve $F+C_{1}$ to $F+O_{1}$. Also, it is in consistent to the simulation result in the previous theoretical model, if the distribution of users are on a line parallel to all the antenna arrays, as shown in curve $\mathrm{O}_{1}+\mathrm{O}_{2}$, the condition number improves significantly, only a few $d B$ away to the reference curve i.i.d. channel. The improvement is very attractive, as the curve chamber $O_{1}+O_{2}$ is even better than most of the curves in the indoor corridor scenario. This suggests that in the near field, a proper relative position of users and antenna arrays can achieve a high spectral efficiency even without rich scatters. However, a deployment of distributed antenna array can fully avoid the worst case that all UEs are located on the normal line of the antenna panel. A surprising finding in the scenario of indoor corridor, though curve $\mathrm{F}+\mathrm{O}_{2}$ is inferior to curve $\mathrm{F}+\mathrm{C}_{2}$, when combining the array $\mathrm{O}_{1}+\mathrm{O}_{2}$, it becomes the curve with the best performance.

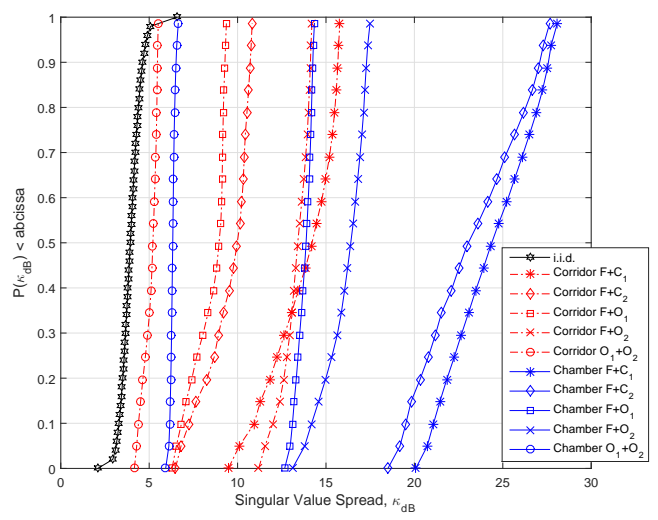

Fig. 7. Comparison of condition number in logarithmic units in CDF. A reference curve of i.i.d. channels with same setting $M=64$ and $K=6$ is included.

We then focus on the correlation coefficients between UEs and highlight the cases of $F+C_{1}$ and $F+O_{1}$ for the ergodic mean of the correlation coefficient of all subcarriers and measurement snapshots. From Fig. 8, for $F+C_{1}$ in the anechoic chamber, we notice that $\mathrm{UE}_{1}$ has lower correlation to its neighbors, however, starting from $\mathrm{UE}_{3}$, its correlation to other neighbors increases to more than 0.8. Also, the correlation coefficients to $\mathrm{UE}_{1}$ and to $\mathrm{UE}_{6}$ are asymmetric, as the UEs are distributed in the direction approaching the farfield of the antenna array, it is more challenging for collocated antenna arrays to separate the UEs. On the other hand, as the direction of the slave array panel $O_{1}$ is parallel to the user distribution, the correlations of users in general decrease greatly. However, when comparing chamber and corridor, the correlation of $\mathrm{UE}_{1}$ to its neighbors increases slightly. The degradation is caused by the fact that now only half the number of antennas are located close to $\mathrm{UE}_{1}$ when compared to the collocated case. Moving to Fig. 9, we show the correlation coefficient of the combination of $\mathrm{O}_{1}+\mathrm{O}_{2}$ in both indoor corridor and chamber. Again, the correlations among all the UEs degrade even more dramatically and are in line with the simulation result in Fig. 3. However, the relateive distribution of users with respect to to the direction of the antenna array is unpredictable, and a distributed antenna array can enjoy the diversity gain.

Finally, we look into the overall performance of ZF sumrates as shown in Fig. 10. We include again the i.i.d. channel for benchmarking and do a joint comparison for all the 


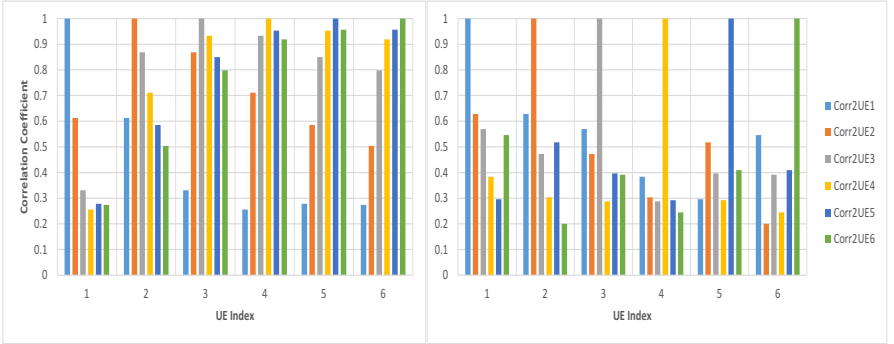

Fig. 8. Correlation coefficient of anechoic chamber (left) collocation of antenna arrays $F+C_{1}$ (right) distributed antenna arrays $F+O_{1}$. An example to express the figure, when fixing the UE index to 1 and so focus on the first column, the pair-wise cross-correlations to the other UEs are shown in different colors in that column.

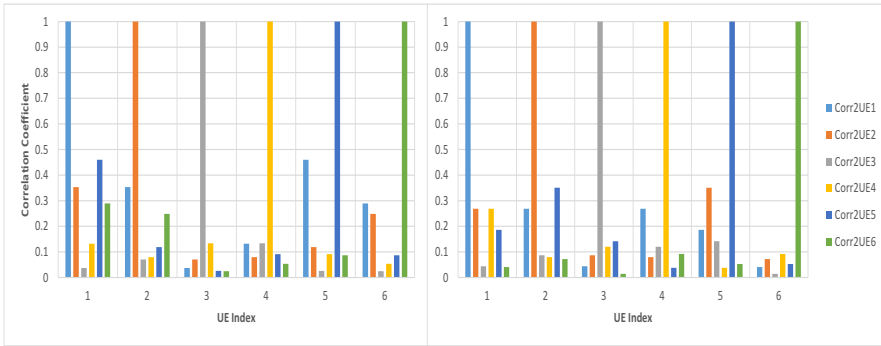

Fig. 9. Correlation coefficient of (left) anechoic chamber antenna arrays $\mathrm{O}_{1}+$ $\mathrm{O}_{2}$ (right) indoor corridor antenna arrays $\mathrm{O}_{1}+\mathrm{O}_{2}$.

measured scenarios for several target levels of $\rho$. For moderate $\rho=15 \mathrm{~dB}, 93 \%(27.5 \mathrm{bits} / \mathrm{s} / \mathrm{Hz})$ of the i.i.d. channel capacity is reached of the indoor corridor scenario $F+O_{1}$, while there is only $76 \%(22.5 \mathrm{bits} / \mathrm{s} / \mathrm{Hz})$ achieved in corridor scenario $F+C_{1}\left(19 \%\right.$ improvement of $F+O_{1}$ to $\left.F+C_{1}\right)$. If we consider a non-scattering environment, for the same SNR level, there is around $140 \%$ improvement of capacity in chamber $F+O_{1}$ $(23.0 \mathrm{bits} / \mathrm{s} / \mathrm{Hz})$ to $F+C_{1}(9.5 \mathrm{bits} / \mathrm{s} / \mathrm{Hz})$. An interesting finding is that the sum-rates in a non-scattering environment $F+O_{1}$ is comparable and even better than the collocated case $F+C_{1}$ in the corridor. This shows that there can be a huge benefit from well distributed antenna arrays for closely located users.

\section{CONCLUSIONS}

In this paper, we investigated user separation of a massive MIMO system in an indoor near-field scenario. The special focus is on the benefits of very simple distributed arrays. For a non-scattering environment with pure LoS, up to $140 \%$ capacity improvement for an orthogonally distributed antenna array case $F+O_{1}$ is measured compared to a traditional parallel collocated case $F+C_{1}$. The contribution from the sub-array in position $O_{1}$ can be understood from the saturation region analysis with the spherical wave channel model. From the model, we learned that the antenna array has difficulties to differentiate users which lie on a normal line to the antenna panel. Instead, if we distribute the sub-array parallel to the line where users are located, the enriched AoAs enhance the user separation. As expected, the benefits in $\mathrm{ZF}$ sum-rates of the best distributed case that we measured, is reduced to $19 \%$ in a rich-scattering environment. This is because the multipaths introduced by scatterers enhance the decorrelation capability of the antenna array and thus a collocated array also works well. In general, the distributed antenna array helps to differentiate closely located users in both measured scenarios. In future work, we will extend our analysis to also include the far field region, and non-LoS scenarios.

\section{ACKNOWLEDGEMENTS}

The authors would like to thank NI engineers Dr. Amal Ekbal and Dr. Jörg Hofrichter for their technical supports of the Massive MIMO code extension.

\section{REFERENCES}

[1] T. L. Marzetta, "Noncooperative cellular wireless with unlimited numbers of base station antennas," Wireless Communications, IEEE Transactions on, vol. 9, no. 11, pp. 3590-3600, November 2010.

[2] F. Rusek, D. Persson, B. K. Lau, E. G. Larsson, T. L. Marzetta, O. Edfors, and F. Tufvesson, "Scaling up MIMO: Opportunities and challenges with very large arrays," IEEE Signal Process. Mag., vol. 30, pp. 40-60, Jan. 2013.

[3] E. G. Larsson, F. Tufvesson, O. Edfors, and T. L. Marzetta, "Massive MIMO for next generation wireless systems," IEEE Commun. Mag., vol. 52, pp. 186-195, Feb. 2014.

[4] J. Flordelis, X. Gao, G. Dahman, F. Rusek, O. Edfors, and F. Tufvesson, "Spatial separation of closely-spaced users in measured massive multiuser MIMO channels," in Proc. IEEE ICC, June 2015.

[5] S. Payami and F. Tufvesson, "Channel measurements and analysis for very large array systems at 2.6 GHz," in 2012 6th European Conference on Antennas and Propagation (EUCAP), Mar. 2012, pp. 433-437.

[6] M. Gauget et al., "Channel measurements with different antenna array geometries for massive MIMO systems," in Proc. Int. ITG Conf. Syst., Commun. Coding (SCC), Feb. 2015, pp. 1-6.

[7] A. Paulraj, R. Nabar, and D. Gore, Introduction to Space-Time Wireless Communications. The Edinburg Building, Cambridge CB2 8RU, UK: Cambridge University Press, first ed., 2008.

[8] H. Q. Ngo, E. Larsson, and T.Marzetta, "Aspects of favorable propagation in massive MIMO," in Proc. 22nd EUSIPCO, Sep. 2014, pp. 76-80.

[9] LabVIEW Communications 802.11 Application Framework White Paper. (2015, October 06). Retrieved ] from http://www.ni.com/productdocumentation/52533/en/

[10] J.-S. Jiang and M. A. Ingram, "Spherical wave model for short-range MIMO," IEEE Trans. Commun., vol. 53, no. 9, pp. 1534 - 1541, Sept. 2005.

[11] S. Vishwanath, N. Jindal, and A. Goldsmith, "Duality, achievable rates, and sum-rate capacity of MIMO broadcast channels," IEEE Trans. Inf. Theory, vol. 49, pp. 2658-2668, Oct. 2003. 